\title{
What Went Wrong? Forensics \& Food Microstructural Characterization
}

Var L. St. Jeor

Dept. of Ingredient, Material, Nutrition; Cargill Incorporated; Plymouth, MN, USA

When we think of food, we often think in terms of appearance, texture, color, aroma and taste. We can think of these as "primary traits." But from a commercial and scientific point of view there are other important factors that are more difficult to qualify, all of which can have impact on the food in positive and negatives ways. Therefore, they also have impact on the food's primary traits. We can classify these secondary traits as "microstructure." For this discussion, "nanostructure" is also a component of microstructure.

When microstructure is right, we really don't think much about it. The food is behaving as it should. But when the quality of the food is off, it is often because the microstructure is wrong. Because microstructure is usually on a micro-to-nano scale, it can be challenging to find ways to analyze it, quantify it and reproduce it; all of which are key to being able to reproduce the food correctly on a commercial scale. And that is key to having a consistent product.

One aspect of food forensics can be thought of as understanding correct microstructure so we can apply that knowledge when things aren't right, allowing us to define what is wrong. Once we can define, qualify and quantify what is wrong, that allows for corrections to be made.

There are many tools in the forensics arsenal that allow us to analyze microstructure, and imaging is one way to investigate these faults. It allows us to see what went wrong. Since microstructure is usually on a scale of micro-to-nano, some sort of microscopy is needed to do the imaging. But much of microstructure is not obvious without sample preparation designed to reveal the specific microstructure in question.

Since food forensics has not been a specific field of study until fairly recently, the problem was in finding methods of sample preparation that would be appropriate too food. The answer to discovering or inventing these methods came when food started being thought of as more than food. For example, food is a biological, either botanical or zoological. But food is also a chemical, and it is a material. As such, all of the methods available to these other fields of study become available to foods.

For example, various gums are often added to a food to aid in microstructure maintenance and stabilization. When present in a food gums are usually not recognizable, blending-in with all the other ingredients. But, if the food primary properties are off, how does one know what is causing it? How would one know it was the gum that is not functioning properly? Using biologically-based histology or cytology techniques, in the form of microtomy and/or specific stains ${ }^{1}$ that are unique to gum, provide a specific color to identify the gum. That can be the imaging aid that allows microscopy to do the needed forensics analysis. The trick comes in modifying those biological methods to be adaptable to the food at hand. 
References:

[1] P Gray, "Handbook of Basic Microtechnique," $3^{\text {rd }}$ ed., (1964), McGraw Hill, Inc.

[2] FJ Green (ed), The Sigma-Aldrich Handbook of Stains, Dyes \& Indicators, Aldrich Chemical Co. (1990).
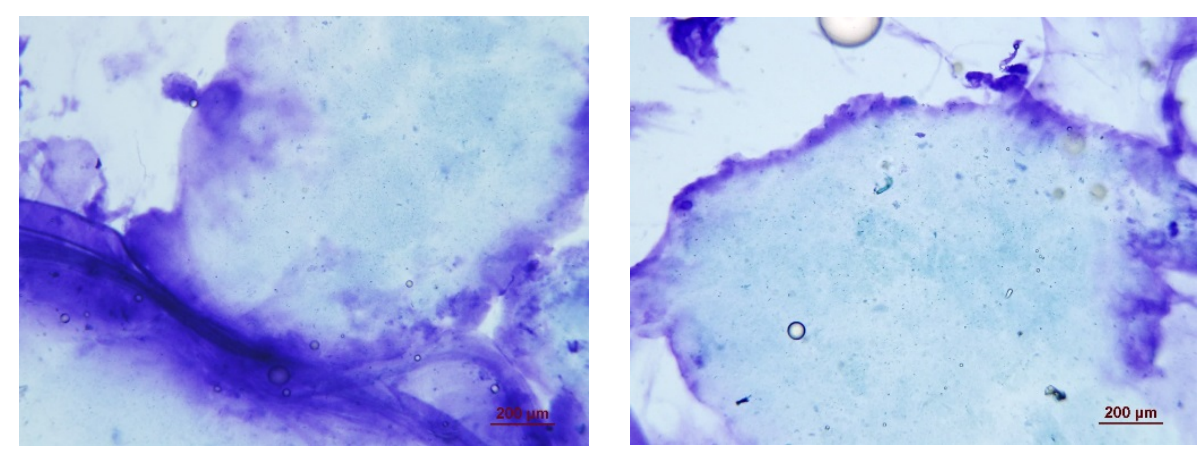

Figure 1. Light microscope images of a soup containing many ingredients. A concern was for "gel balls" found within the soup. Staining with Methylene Blue (a metachromatic stain) allows us to identify gum (purple) and protein (blue). In this case, a gum that is not fully dispersed in the soup has entangled protein, also not fully dispersed. Gum-encapsulated protein was the cause for the gel balls.
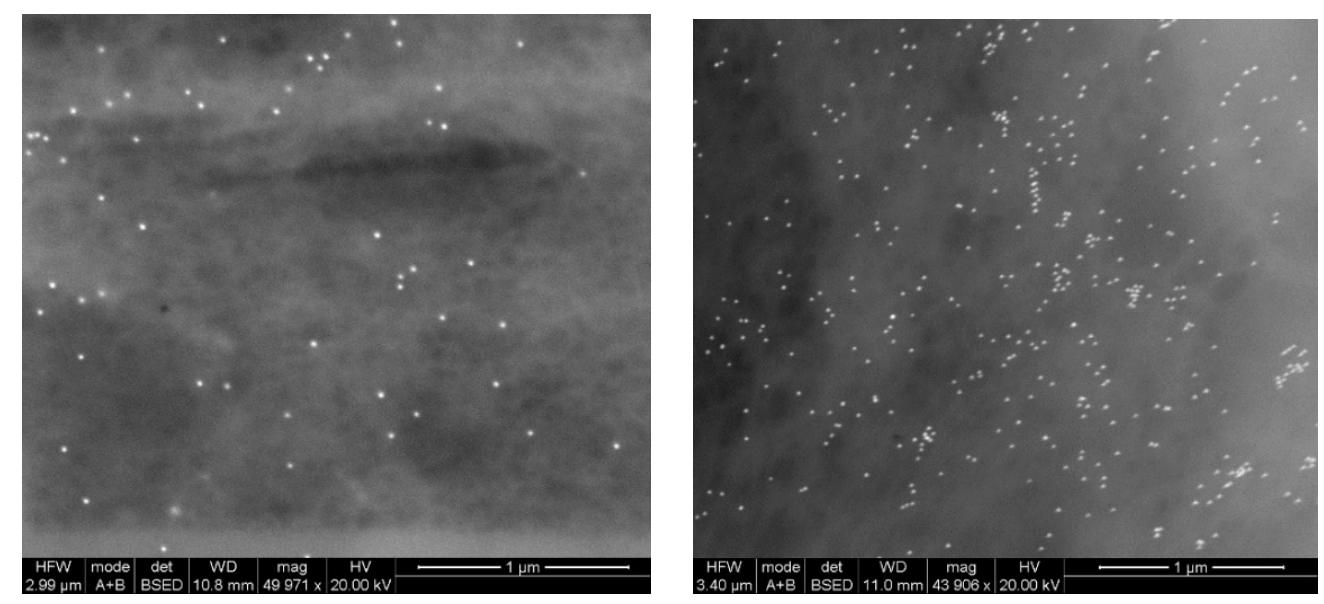

Figure 2. Cellulous samples displaying different functional properties were treated using enzymecytochemistry to determine molecular structural differences. This is something akin to immunocytochemistry, but with an enzyme (cellulase) in place of an antibody. The enzyme is functioning much like an antibody label would. Cellulase was conjugated with $20 \mathrm{~nm}$ gold particles using an immuno-like procedure to create the label. Treatment of the cellulose allowed the label to attach to the aldehyde group of a broken glucose chain molecule. The right sample is displaying more molecular damage, as indicated by an increase in the number of attached gold particles. Documentation was done by E-SEM in backscatter mode to enhance gold visibility. 